\title{
On the Tails of the Distribution of the Sum of Lognormals
}

\author{
Sebastian S. Szyszkowicz, Student Member, IEEE, Halim Yanikomeroglu, Member, IEEE
}

\begin{abstract}
Finding the distribution of the sum of lognormal random variables is an important mathematical problem in wireless communications, as well as in many other fields. While several methods exist to approximate this distribution, their performance tends to deteriorate in both tail areas. Finding a good overall fit remains an open problem. Other disadvantages of these methods are their complexity and, in some cases, their limitation to particular scenarios.

In this paper we examine the sum of independent lognormal random variables with arbitrary parameters. We define the concept of best lognormal fit to a tail and show what it means in terms of convergence. We restate a known result about asymptotes to the higher tail of the distribution. To our knowledge, the lower tail has not yet been studied. We give a simple closed-form expression for an asymptote to the lower tail.

We also show that known methods for finding the sum of lognormals use distribution functions that do not have this asymptotic behaviour in the tails. Our results are complementary to the existing knowledge, which together can combine to solve the problem of the sum of lognormals simply and exactly. We support our results by simulations.
\end{abstract}

Index Terms-interference statistics, sum of lognormals, tail distribution.

\section{INTRODUCTION}

$\mathrm{T}$ HE problem of the sum of lognormals (SLN) arises in wireless communications when the distribution of the total interference power coming from several shadowed sources is required. It is of significant interest, as it has been explored for some 50 years [1]-[3], and has received renewed interest in the wireless community in the past few years [4]-[12]. It also arises in economics [13], physics [14], electronics [15], and is of interest to the statistical community [16]. In general, the lognormal summands need not be independent and identically distributed (i.i.d.). Indeed, the total interference power in a cellular system is composed of both near and far-away interferers, and the shadowing paths may be correlated according to the angle of arrival. Thus, summands of different marginal distributions and with possible correlation among

Manuscript received September 28, 2006. This work was supported in part by an award from the Natural Sciences and Engineering Research Council (NSERC) of Canada, and by Wireless Technology Labs, Nortel Networks, Ottawa.

S. S. Szyszkowicz is with the Broadband Communications and Wireless Systems (BCWS) Centre, Dept. of Systems and Computer Engineering, Carleton University, Ottawa, ON K1S 5B6, Canada (phone: 613-520-2600 x1948, fax: 613-520-5727, e-mail: sz@ sce.carleton.ca)

H. Yanikomeroglu is also with BCWS (e-mail: halim@sce.carleton.ca). them should be considered.

The problem can be mathematically stated as follows: Let

$$
X=\sum_{i=1}^{N} Y_{i}
$$

We assume in this paper that the summands $Y_{i}$ are independent. The probability density function $(p d f)$ of each summand is lognormal:

$$
Y_{i} \sim f_{i}(x)=\frac{1}{\sqrt{2 \pi} x \sigma_{i}} e^{-\frac{1}{2}\left(\frac{\ln x-\mu_{i}}{\sigma_{i}}\right)^{2}}, x>0 .
$$

The cumulative distribution function $(c d f)$ is:

$$
F_{i}(x)=\Phi\left(\frac{\ln x-\mu_{i}}{\sigma_{i}}\right), x>0,
$$

where $\Phi(x)$ is the standard normal $c d f$. Then

$$
X \sim f(x)=\frac{\partial}{\partial x} F(x), x>0
$$

follows the SLN distribution, with no known closed form. When the summands $Y_{i}$ are independent, $f(x)$ is the convolution of all $f_{i}(x)$.

There exist various approximate solutions to this distribution, which generally consist of two distinct parts: choosing a general form for the SLN distribution, and obtaining its parameters for particular cases. One approach has been to approximate the SLN distribution by a lognormal, and various methods were used to find the best-fitting lognormal parameters [1],[3]-[6]. However, it has become clear that as the number of summands increases, the SLN distribution appears less and less lognormal [7]. Indeed, by virtue of the Central Limit Theorem, the SLN approaches a normal distribution, but this convergence is very slow [14]. There also exist other methods, which use more complex distribution functions with three or more free parameters [7]-[10]. Nonparametric solutions have also been proposed: piecewiselognormal [11], or product-of-lognormal [12]. Each of these methods has its drawbacks: the first class of methods, where a lognormal approximation is used, cannot possibly be accurate over the entire range of $x$. Many methods require numerical integration to find the required parameters [3]-[5], [8], [10], [11]. While the behaviour of the upper tail, i.e. as $x \rightarrow \infty$ is known [2], [16], that of the lower tail, i.e. as $x \rightarrow 0$ has no simple expression. Some methods have only been verified for the case of i.i.d. summands [7], [9]. Also, we will show that methods that match the body of the distribution well [7]-[10], 
are not necessarily accurate in the far tails. On the other hand, the piecewise-lognormal method proposed in [11] does give a good behaviour at the tails, but again requires numerical integration and does not perform as well in the body. Hence, there is no single method that really gives a complete solution to the problem, and there is no closed form for the lower tail.

This paper intends to cover both tails of the SLN distribution. We first define what we mean for a distribution to have a best lognormal fit at a given tail. We use this definition throughout the paper to compare tail behaviours. We then restate known results on the upper tail. Our main contribution is to give a simple approximation for the lower tail. We then show that while both tails of the SLN have a best lognormal fit, those of all the recently proposed distributions [7]-[10] do not and thus cannot possibly give good fits in either tail. We support our results by simulations, showing that methods [7][9] diverge and our approximations converge at both tails. We conclude that there exist simple expressions for the tails of the SLN, and that it would be interesting to search for SLN distributions that have tails of the same form.

\section{TAIL PROPERTIES OF THE SUM OF LOGNORMALS}

\section{A. Tail Properties on Lognormal Paper}

It is convenient to look at the $c d f$ of the SLN on lognormal probability paper [4], where lognormal distributions are mapped onto straight lines by the following transformation $T$ :

$$
T: G(x) \mapsto \tilde{G}(x)=\Phi^{-1}\left(G\left(e^{x}\right)\right) .
$$

Indeed this transformation maps:

$$
T: \Phi\left(\frac{\ln x-\mu_{i}}{\sigma_{i}}\right) \mapsto \frac{x-\mu_{i}}{\sigma_{i}},
$$

which is a straight line of strictly positive slope. Conversely, each line of strictly positive slope corresponds to a unique lognormal distribution.

It is therefore useful to work in this domain: we plot our simulated curves, the proposed asymptotes, and the $c d f$ curves obtained by other methods on lognormal paper. Before proceeding, we show how convergence in the transformed domain relates to convergence in the linear domain.

Definition:

Let $T: F(x) \mapsto \tilde{F}(x)$. Then $\frac{x-\mu}{\sigma}$ is considered an asymptote to $\tilde{F}(x)$ at $l= \pm \infty$ if and only if:

$$
\lim _{x \rightarrow l} \tilde{F}(x)-\frac{x-\mu}{\sigma}=0 .
$$

We say that $F(x)$ has a best lognormal fit $\Phi\left(\frac{\ln x-\mu}{\sigma}\right)$ at $l$.

\section{Theorem 1:}

For any supports $I, J$, such that $x \in I \Leftrightarrow e^{x} \in J$ we have:

$\forall x \in I, \tilde{F}(x)>\frac{x-\mu}{\sigma} \Leftrightarrow \forall x \in J, F(x)>\Phi\left(\frac{\ln x-\mu}{\sigma}\right)$, and

$\forall x \in I, \tilde{F}(x)<\frac{x-\mu}{\sigma} \Leftrightarrow \forall x \in J, F(x)<\Phi\left(\frac{\ln x-\mu}{\sigma}\right)$.

Proof:

Let $g(x)=\tilde{F}(x)-\frac{x-\mu}{\sigma}>0$ and

$T: \Phi\left(\frac{\ln x-\mu}{\sigma}+g(\ln x)\right)=F(x) \mapsto \tilde{F}(x)=\frac{x-\mu}{\sigma}+g(x)$.

Since $\forall x \in I, g(x)>0 \Leftrightarrow \forall x \in J, g(\ln x)>0$ and $\Phi(x)$ is strictly increasing, we have $\forall x \in J, F(x)>\Phi\left(\frac{\ln x-\mu}{\sigma}\right)$, which proves (8). The proof is analogous for (9).

Theorem 2:

If $\lim _{x \rightarrow-\infty} \tilde{F}(x)-\frac{x-\mu}{\sigma} \neq 0$ or does not exist, then

$$
\lim _{x \rightarrow 0} \frac{F(x)}{\Phi\left(\frac{\ln x-\mu}{\sigma}\right)} \in\{0,+\infty\} \text { or does not exist. }
$$

Likewise, if $\lim _{x \rightarrow+\infty} \tilde{F}(x)-\frac{x-\mu}{\sigma} \neq 0$ or does not exist, then

$$
\lim _{x \rightarrow+\infty} \frac{1-F(x)}{1-\Phi\left(\frac{\ln x-\mu}{\sigma}\right)} \in\{0,+\infty\} \text { or does not exist. }
$$

Proof:

If $\lim _{x \rightarrow-\infty} \tilde{F}(x)-\frac{x-\mu}{\sigma} \neq 0$ or does not exist, then

$$
\begin{gathered}
\forall \varepsilon>0, \forall \delta, \exists x<\delta,\left|\tilde{F}(x)-\frac{x-\mu}{\sigma}\right| \geq \varepsilon \\
\Leftrightarrow \forall \varepsilon>0, \forall \delta, \exists x<\delta, \\
\tilde{F}(x) \geq \frac{x-(\mu-\sigma \varepsilon)}{\sigma} \vee \tilde{F}(x) \leq \frac{x-(\mu+\sigma \varepsilon)}{\sigma}
\end{gathered}
$$

Applying our Theorem 1:

$$
\begin{aligned}
\forall \varepsilon>0, \forall \delta, \exists x<\delta, \tilde{F}(x) \geq \frac{x-(\mu-\sigma \varepsilon)}{\sigma} \\
\Leftrightarrow \forall \varepsilon>0, \forall \delta, \exists x<e^{\delta}, F(x) \geq \Phi\left(\frac{\ln x-(\mu-\sigma \varepsilon)}{\sigma}\right) \\
\Rightarrow \lim _{x \rightarrow 0} \frac{F(x)}{\Phi\left(\frac{\ln x-\mu}{\sigma}\right)} \geq \lim _{x \rightarrow 0} \frac{\Phi\left(\frac{\ln x-(\mu-\sigma \varepsilon)}{\sigma}\right)}{\Phi\left(\frac{\ln x-\mu}{\sigma}\right)} \\
=\lim _{x \rightarrow 0} e^{-\frac{1}{2} \varepsilon^{2}-\frac{\ln x-\mu}{\sigma} \varepsilon}=+\infty .
\end{aligned}
$$

Likewise:

$$
\forall \varepsilon>0, \forall \delta, \exists x<\delta, \tilde{F}(x) \leq \frac{x-(\mu+\sigma \varepsilon)}{\sigma}
$$

$\Leftrightarrow \forall \varepsilon>0, \forall \delta, \exists x<e^{\delta}, F(x) \leq \Phi\left(\frac{\ln x-(\mu+\sigma \varepsilon)}{\sigma}\right)$ 


$$
\begin{gathered}
\Rightarrow \lim _{x \rightarrow 0} \frac{F(x)}{\Phi\left(\frac{\ln x-\mu}{\sigma}\right)} \leq \lim _{x \rightarrow 0} \frac{\Phi\left(\frac{\ln x-(\mu+\sigma \varepsilon)}{\sigma}\right)}{\Phi\left(\frac{\ln x-\mu}{\sigma}\right)} \\
=\lim _{x \rightarrow 0} e^{-\frac{1}{2} \varepsilon^{2}+\frac{\ln x-\mu}{\sigma} \varepsilon}=0 . \\
\text { But } \forall x, \frac{F(x)}{\Phi\left(\frac{\ln x-\mu}{\sigma}\right)} \geq 0, \text { thus: } \\
\quad \forall \varepsilon>0, \forall \delta, \exists x<\delta, \tilde{F}(x)<\frac{x-(\mu+\sigma \varepsilon)}{\sigma} \\
\Rightarrow \lim _{x \rightarrow 0} \frac{F(x)}{\Phi\left(\frac{\ln x-\mu}{\sigma}\right)}=0 \text { or does not exist. }
\end{gathered}
$$

Thus, if $\lim _{x \rightarrow-\infty} \tilde{F}(x)-\frac{x-\mu}{\sigma} \neq 0$ or does not exist, then $\lim _{x \rightarrow 0} \frac{F(x)}{\Phi\left(\frac{\ln x-\mu}{\sigma}\right)}$ is $0,+\infty$, or does not exist. The proof for

(11) is analogous. This proves Theorem 2.

\section{Conclusion:}

The first theorem says that inequalities are conserved under the lognormal transformation $T$. The second theorem implies that if, on lognormal paper, a line $L$ is not the best lognormal fit to a curve $C$ at the limit $l$, then the corresponding distributions have tails in the corresponding limit $e^{l}$ that are not comparable, i.e. their ratio will diverge. In other words, a necessary (but not sufficient) condition for approximating a distribution by a lognormal at a given tail is that the two distributions be asymptotes to each other on lognormal paper.

\section{B. The Upper Tail Asymptote}

The behaviour of the SLN distribution $F(x)$ as $x \rightarrow+\infty$ has been previously studied [2], [16]. It was shown in [2] that the moments $E\left\{X^{n}\right\}_{\text {of a }}$ SLN are dominated by the heaviest summand(s) as $n$ increases. In [11], it is suggested that matching higher and higher moments of the sum to those of a lognormal distribution gives a better and better lognormal asymptote to the upper tail behaviour. In [17], it is argued that moments of positive random variables (as is our case) determine the upper tail. All this information suggests that looking at the heaviest summand(s) alone will give us a good approximation of the tail. Reference [16] gives a simple procedure to find the upper tail asymptote:

1. Choose the summands with the highest logarithmic standard deviation:

$$
\sigma_{U}=\max _{i=1, \ldots, N} \sigma_{i} .
$$

2. Among these summands, chose those with the highest logarithmic mean:

$$
\mu_{U}=\max _{i: \sigma_{i}=\sigma_{U}} \mu_{i}
$$

3. Count the number of these "heaviest" summands:

$$
\kappa_{U}=\#\left\{\left(\mu_{i}, \sigma_{i}\right)=\left(\mu_{U}, \sigma_{U}\right)\right\},
$$

where $\#\{S\}$ is the cardinality of set $S$.

The tail distribution is then that of the heaviest summand(s), multiplied by the number of these heaviest summands:

$$
\begin{gathered}
f(x)^{\stackrel{x \rightarrow+\infty}{\sim}} f_{U}(x)=\frac{\kappa_{U}}{\sqrt{2 \pi} x \sigma_{U}} e^{-\frac{1}{2}\left(\frac{\ln x-\mu_{U}}{\sigma_{U}}\right)^{2}} \\
F(x)^{\stackrel{x \rightarrow+\infty}{\sim}} F_{U}(x)=\kappa_{U} \Phi\left(\frac{\ln x-\mu_{U}}{\sigma_{U}}\right)+\left(1-\kappa_{U}\right) .
\end{gathered}
$$

Since the distribution is a finite constant multiple of a lognormal, our Theorem 2 says that the SLN distribution will have a best lognormal fit $\Phi\left(\frac{x-\mu_{U}}{\sigma_{U}}\right)$ at $+\infty$.

Note that [16] proves that this result is also valid when the summands with the same marginal distributions are correlated.

It is argued in [16] that if a summand is not heaviest, but nearly so, it also contributes significantly to the tail for large but bounded $x$. Thus, a better asymptote is the distribution of the maximum of the summands:

$$
F(x) \stackrel{x \rightarrow \infty}{\sim} F_{\max }(x)=\prod_{i} \Phi\left(\frac{\ln x-\mu_{i}}{\sigma_{i}}\right) .
$$

This formula is an upper bound, which corresponds to the lower bound (in the complementary domain) given in [12], which is tight for high values of $x$.

\section{The Lower Tail Asymptote}

To our knowledge, there is no work dealing directly with the lower tail behaviour of the SLN. There is some literature on the lower tail of the distribution of the sum of positive random variables, but it does not readily apply to the sum of lognormals. The polynomial conditions required in [18] are not fulfilled by the lognormal distribution.

We present here a new result, which is the main contribution of this paper: what we believe to be the best lognormal fit (as defined in section II - A) to the lower tail of the SLN.

Hypothesis:

The SLN has a best lognormal fit $\Phi\left(\frac{x-\mu_{U}}{\sigma_{U}}\right)$ at $-\infty$, where:

$$
\sigma_{L}=\left(\sum_{i=1}^{N} \frac{1}{\sigma_{i}^{2}}\right)^{-\frac{1}{2}}, \quad \mu_{L}=\ln N+\sigma_{L}^{2} \sum_{i=1}^{N} \frac{\mu_{i}}{\sigma_{i}^{2}}
$$

We give an argument for this result in the Appendix. We verify this asymptote by simulation.

\section{TAILS OF SOME PROPOSED DistRibutions THAT APPROXIMATE SLN}

We examine four distributions that have recently been proposed as approximations to the SLN. For each of these we show that they cannot possibly give a good approximation at the tails. We do this formally by showing for all four methods that for the proposed $c d f F_{X X}(x), x \geq 0$, for any $\sigma>0, \mu$, the 
result of the transformation $T: F_{X X}(x) \mapsto \widetilde{F}_{X X}(x)$ cannot have $\frac{x-\mu}{\sigma}$ as an asymptote at $l= \pm \infty$, except in some trivial cases when $F_{X X}(x)$ is lognormal.

We also show that only some of the proposed functions have the lognormal distribution as a special case, which should be a characteristic of an SLN approximation.

We use the constant $\lambda=\frac{\ln 10}{10}$ to convert from decibel units.

\section{A. Beaulieu-Rajwani (BR) Method [7]}

This approximating $c d f$ has three parameters $\left(a_{0}, a_{1}, a_{2}\right)$ :

$$
\begin{gathered}
F_{B R}(x)=\Phi\left(a_{0}-a_{1} x^{-\frac{a_{2}}{\lambda}}\right), x \geq 0, a_{0}>0, a_{1}>0, a_{2}>0, \\
T: F_{B R}(x) \mapsto \tilde{F}_{B R}(x)=a_{0}-a_{1} e^{-\frac{a_{2}}{\lambda} x} .
\end{gathered}
$$

Then:

$$
\lim _{x \rightarrow l} \tilde{F}_{B R}(x)-\frac{x-\mu}{\sigma}=a_{0}+\frac{\mu}{\sigma}-\lim _{x \rightarrow l} a_{1} e^{-\frac{a_{2}}{\lambda} x}+\frac{x}{\sigma}= \pm \infty .
$$

Hence, the BR distribution does not have a best lognormal fit at either tail.

In [9], it is mentioned that this form does not include the lognormal distribution as a special case. We can, however, obtain convergence of $F_{B R}(x) \rightarrow \Phi\left(\frac{\ln x-\mu}{\sigma}\right) \forall x$ :

Let us consider $\mu, \sigma$ as constants, such that:

$$
\mu=\frac{a_{1}-a_{0}}{a_{1} a_{2}}, \sigma=\frac{1}{a_{1} a_{2}} .
$$

Then

$$
F_{B R}(x)=\Phi\left(\frac{-\frac{a_{1}-a_{0}}{a_{1} a_{2}}-\left(\frac{x^{-a_{2}}-1}{a_{2}}\right)}{\frac{1}{a_{1} a_{2}}}\right)=\Phi\left(\frac{\left(\frac{1-x^{-a_{2}}}{a_{2}}\right)-\mu}{\sigma}\right) .
$$

Using an alternative definition of the natural logarithm:

$$
\ln x=\lim _{a \rightarrow 0} \frac{1-x^{-a}}{a}, x \geq 0
$$

we obtain:

$$
F_{B R}(x) \stackrel{a_{2} \rightarrow 0}{\longrightarrow} \Phi\left(\frac{\ln x-\mu}{\sigma}\right), x \geq 0 .
$$

Thus, in the limiting case:

$$
a_{2} \rightarrow 0, a_{1}=\left(a_{2} \sigma\right)^{-1}, a_{0}=a_{1}-\mu / \sigma
$$

the $\mathrm{BR}$ distribution is lognormal.

\section{B. Le-Ngoc-Lam (LL) Method [8]}

This approximating $c d f$ has three parameters $(\alpha, \beta, \delta)$ :

$$
F_{L L}(x)=\frac{1}{\Gamma(\alpha)} \gamma\left(\frac{\ln x}{\lambda \beta}-\frac{\delta}{\beta}, \alpha\right), x>e^{\delta \lambda}, \beta>0, \alpha>0,
$$

where $\gamma(x, \alpha)=\int_{0}^{x} e^{-t} t^{\alpha-1} d t$ is the (lower) incomplete gamma function, and $\Gamma(\alpha)$ is the gamma function.
$T: F_{L L}(x) \mapsto \widetilde{F}_{L L}(x)=\Phi^{-1}\left(\frac{1}{\Gamma(\alpha)} \gamma\left(\frac{x-\delta \lambda}{\lambda \beta}, \alpha\right)\right), x>\delta \lambda$.

The function is not even defined for arbitrarily low values.

In the upper tail, we use an approximation of the incomplete gamma function [19]:

$$
\Gamma(\alpha)-\gamma(x, \alpha) \stackrel{x \rightarrow \infty}{\sim} x^{\alpha-1} e^{-x}\left(1+O\left(x^{-1}\right)\right),
$$

and an approximation to the inverse standard normal $c d f$ [20]:

$$
\Phi^{-1}(x) \stackrel{x \rightarrow 0}{\sim}-\sqrt{-2 \ln (2 \sqrt{\pi} x \sqrt{-\ln (2 x)})} .
$$

Then:

$$
\begin{gathered}
\lim _{x \rightarrow+\infty} \tilde{F}_{L L}(x)-\frac{x-\mu}{\sigma} \\
=\frac{\mu-\lambda \delta}{\sigma}-\lim _{x \rightarrow+\infty} \Phi^{-1}\left(\frac{1}{\Gamma(\alpha)} x^{\alpha-1} e^{-x}\right)-\frac{\lambda \beta}{\sigma} x \\
=\lim _{x \rightarrow+\infty} \sqrt{2 x}-\frac{\lambda \beta}{\sigma} x+\frac{\mu-\lambda \delta}{\sigma}=-\infty .
\end{gathered}
$$

Hence, the LL distribution does not have a best lognormal fit at either tail.

\section{Zhao - Ding (ZD) Method [9]}

This approximating $c d f$ has three parameters $\left(c_{0}, c_{1}, c_{2}\right)$ :

$$
\begin{gathered}
F_{Z D}(x)=\Phi\left(c_{0}+c_{1} \frac{\ln x}{\lambda}+c_{2}\left(\frac{\ln x}{\lambda}\right)^{2}\right), x \geq 0, \\
T: F_{Z D}(x) \mapsto \tilde{F}_{Z D}(x)=c_{0}+\frac{c_{1}}{\lambda} x+\frac{c_{2}}{\lambda^{2}} x^{2} .
\end{gathered}
$$

Then, if $c_{2} \neq 0$ :

$$
\begin{gathered}
\lim _{x \rightarrow l} \tilde{F}_{Z D}(x)-\frac{x-\mu}{\sigma} \\
=\frac{c_{2}}{\lambda^{2}} x^{2}+\left(\frac{c_{1}}{\lambda}-\frac{1}{\sigma}\right) x+c_{0}+\frac{\mu}{\sigma}= \pm \infty .
\end{gathered}
$$

Hence, the ZD distribution does not have a best lognormal fit at either tail, except in the lognormal case $c_{2}=0$.

\section{Zhang-Song (ZS) Method [10]}

The proposed distribution for the SLN in $\mathrm{dB}$ is Pearson type IV, which has four parameters $(u, m, d, v)$. Consequently, the $p d f$ of the SLN in linear scale has distribution:

$$
\begin{gathered}
f_{Z S}(x)=\frac{k}{\lambda x}\left(1+y^{2}\right)^{-m} e^{-v \arctan y}, \\
y=\frac{\ln x}{\lambda d}+\frac{u}{d}, m>\frac{1}{2}, x \geq 0,
\end{gathered}
$$

and $k$ is the constant chosen so that the pdf be valid and is found by numerical integration for each particular case. It would seem that the $c d f$ is not readily available in closed form. However, we can still study its tail behaviour:

$$
\begin{gathered}
f_{Z S}(x) \stackrel{x \rightarrow 0}{\sim} k|d|^{2 m} \lambda^{2 m-1} x^{-1}|\ln x|^{-2 m} e^{v \frac{\pi}{2} \operatorname{sgn}(d)} \\
=C_{1} \frac{|\ln x|^{-2 m}}{x}, C_{1}>0,
\end{gathered}
$$




$$
F_{Z S}(x) \stackrel{x \rightarrow 0}{\sim}=\int_{0}^{x} C_{1} \frac{|\ln y|^{-2 m}}{y} d y=C_{1} \frac{|\ln x|^{1-2 m}}{2 m-1} .
$$

Again, using the result (34) from [20]:

$$
\begin{aligned}
& T: F_{Z S}(x) \mapsto \tilde{F}_{Z S}(x) \stackrel{x \rightarrow-\infty}{\sim} \Phi^{-1}\left(\frac{C_{1}}{2 m-1}|x|^{1-2 m}\right) \\
& \stackrel{x \rightarrow-\infty}{\sim}-\sqrt{(4 m-2)} \sqrt{\ln (-x)} \Rightarrow \lim _{x \rightarrow-\infty} \tilde{F}_{Z D}(x)-\frac{x-\mu}{\sigma} \\
& \quad=-\lim _{x \rightarrow-\infty} \sqrt{(4 m-2)} \sqrt{\ln (-x)}+\frac{x-\mu}{\sigma}=+\infty .
\end{aligned}
$$

A similar analysis applies for the upper tail. Hence, the ZS distribution does not have a best lognormal fit at either tail.

\section{E. Conclusion about the four methods}

We have seen that none of the proposed functions can have linear asymptotes in the transformed domain. However, since at least the upper tail of the SLN has been proven to have a linear asymptote in the lognormal domain (and we conjecture that the lower tail has the same property), all of the proposed functions will have unbounded relative error in the tails.

\section{SimULATIONS AND COMPARISONS}

We present Monte-Carlo simulation $c d f$ curves of the SLN, which we use as the standard of comparison, and reproduce the curves from methods [7]-[9]. We plot all these on lognormal probability paper, along with the lower asymptotes proposed in this paper.

We use the values from [7, Table I] to obtain curves for the $\mathrm{BR}$ method for several i.i.d. cases. For the LL curves, we used the values from [8, Table I]. Note that in the fifth row of that table, the log-variance should be $12 \mathrm{~dB}$, not $6 \mathrm{~dB}$. For the $\mathrm{ZD}$ curves, we used [9, eq.(18)-(20)], where $\sigma$ is in $\mathrm{dB}$, which are applicable to i.i.d. cases only. We were not able to reproduce the ZS curves, because the $c d f$ is not known.

We also plot the theoretical asymptotes: one straight asymptote, the best lognormal fit, from (22), and one lognormal-times-constant asymptote for each tail, from (45), (46). We see that the simulated curve tends to be sandwiched between these two curves. By virtue of our theorems, this would imply that the lower SLN tail is indeed well approximated by the lognormal with parameters given by (22).

We see that in all cases the BR and LL methods, and for most cases, the ZD method, give accurate results in the body of the distribution. However, it is evident from the figures that each method gives a curve that tends to diverge from the best lognormal fit of the SLN at either tail, the SLN converging instead to the proposed asymptotes.

In [8] it could already be seen that with the LL method one obtains this divergence on lognormal paper in the tails.

\section{CONCLUSION}

There does not exist at this moment any closed form (without numerical integration) method to obtain a good approximation to the SLN distribution. The LL [8] and [ZS] methods approximate the body well and are suited to the general, non i.i.d. case. Since their complexity resides essentially in the numerical computation of the moments of the logarithm of a sum of lognormals, they are essentially improvements on the lognormal fitting method proposed by Schwartz and Yeh [3]. We propose that the much-used method [3] be put aside in favour of [8] or possibly [10].

Our suggestion for continuing work would be to combine the methods for finding the body of the distribution with our results on the tails to obtain an overall closed-form solution.

\section{APPENDIX}

In order to find the lower tail of the SLN distribution, we need to find the convolution near 0 of two lognormal functions. We proceed as follows: let us sample the lognormal function at $0, x, 2 x, \ldots$, where $x$ is small. Then, we perform a discrete convolution and look at the first non-zero sample:

$$
f(2 x) \stackrel{x \rightarrow 0}{\approx} x f_{1}(x) f_{2}(x),
$$

Proceeding likewise for $N$ functions:

$$
\begin{aligned}
& f(N x) \stackrel{x \rightarrow 0}{\approx} x^{N-1} \prod_{i=1}^{N} f_{i}(x)= \\
& \frac{N^{1-N}}{\sqrt{2 \pi}^{N} x \prod_{i=1}^{N} \sigma_{i}} e^{-\frac{1}{2} \ln ^{2} x \sum_{i=1}^{N} \frac{1}{\sigma_{i}^{2}}+\ln x \sum_{i=1}^{N} \frac{\mu_{i}}{\sigma_{i}^{2}}-\frac{1}{2} \sum_{i=1}^{N} \frac{\mu_{i}^{2}}{\sigma_{i}^{2}}} .
\end{aligned}
$$

Consequently:

$$
f(x) \stackrel{x \rightarrow 0}{\approx} \frac{\kappa_{L}}{\sqrt{2 \pi} \sigma_{L} x} e^{-\frac{1}{2}\left(\frac{\ln x-\mu_{L}}{\sigma_{L}}\right)^{2}},
$$

where:

$$
\kappa_{L}=N \sigma_{L}\left(\prod_{i=1}^{N} \sigma_{i}\right)^{-1} \sqrt{2 \pi}^{1-N} e^{\frac{1}{2} \sigma_{L}^{2}\left(\sum_{i=1}^{N} \frac{\mu_{i}}{\sigma_{i}^{2}}\right)^{2}-\frac{1}{2} \sum_{i=1}^{N} \frac{\mu_{i}^{2}}{\sigma_{i}^{2}}}
$$

and $\mu_{L}, \sigma_{L}$ are given by (22).

Thus, assuming the approximation in (43) is good, by Theorem 2, we can conclude that the SLN has best lognormal fit $\Phi\left(\frac{\ln x-\mu_{L}}{\sigma_{L}}\right)$ at 0 . It is important to note that, because the lognormal distribution is very flat near zero, in fact, $\frac{\partial^{n}}{\partial x^{n}} \Phi\left(\frac{\ln x-\mu}{\sigma}\right)=0 \forall n=1,2, \ldots$, the convolution is difficult to analyse, as it does not fit in the framework of [18], nor is there a guarantee that the discrete convolution performed here is very good. Indeed, we find by simulations that the value of $\kappa_{L}$ does not improve the approximation much.

\section{ACKNOWLEDGMENT}

We would like to thank Dr. Eman Fituri and Dr. Shalini Periyalwar, Carleton University and Dr. John Thompson, University of Edinburgh, for their valuable help. 


\section{REFERENCES}

[1] L. Fenton, "The sum of log-normal probability distributions in scatter transmission systems," IEEE Trans. Communications, vol.8, Mar. 1960 pp. 57-67.

[2] W. A. Janos, "Tail of the distribution of sums of lognormal variates," IEEE Trans. Information Theory, vol. IT-16, May 1970, pp. 299-302.

[3] S. C. Schwartz, Y. S. Yeh, "On the distribution function and moments of power sums with lognormal components," Bell Syst. Tech. J., vol. 61, 1982, pp. 1441-1462.

[4] N. C. Beaulieu, Q. Xie, "An optimal lognormal approximation to lognormal sum distributions," IEEE Trans. Vehicular Tech., vol. 53, Mar. 2004, pp. 479 - 489.

[5] J. Wu, N. B. Mehta, J. Zhang, "Flexible lognormal sum approximation method," IEEE GLOBECOM 2005, vol. 6, Nov. 2005, pp. 3413-3417.

[6] M. Pratesi, F. Santiccu, F. Graziosi, "Generalized moment matching for the linear combination of lognormal RVs: application to outage analysis in wireless systems," IEEE Trans. Wireless Communications, vol. 5, May 2006, pp. 1122-1132.

[7] N. C. Beaulieu, F. Rajwani, "Highly accurate simple closed-form approximations to lognormal sum distributions and densities," IEEE Communications Letters, vol.8, Dec. 2004, pp. 709-711.

[8] C. L. J. Lam, T. Le-Ngoc, "Log shifted gamma approximation to lognormal sum distributions," IEEE ICC 2005, vol. 1, May 2005, pp. 495-499

[9] L. Zhao, J. Ding, "A strict approach to approximating lognormal sum distributions", CCECE 2006, May 2006, pp.286.

[10] Q. T. Zhang, S. H. Song, "Model Selection and Estimation for Lognormal Sums in Pearson's Framework," 63rd IEEE VTC 2006Spring, vol. 6, 2006, pp. 2823-2827.

[11] J. C. S. Santos Filho, M. D. Yacoub, P. Cardieri, "Highly accurate range -adaptive lognormal approximation to lognormal sum distributions," IEE Electronics Letters, vol. 42, Mar. 2006, pp. 361-362.

[12] S. Ben Slimane, "Bounds on the distribution of a sum of independent lognormal random variables," IEEE Trans. Communications, vol. 49, June 2001, pp. 975-978.

[13] M. Milevsky, S. Posner, "Asian options, the sum of lognormals and the reciprocal Gamma distribution," Journal of Financial and Quantitative Analysis vol. 33, 1998, pp.409-422.

[14] M. Romeo, V. Da Costa, F. Bardou, "Broad distribution effects in sums of lognormal random variables," The European Physical Journal B, vol. 32, 2003, pp.513-525.

[15] H. Chang, S. S. Sapatnekar, "Full-chip analysis of leakage power under process variations, including spatial correlations," 42nd Аnпиаl ACM IEEE Design Automation Conference, 2005, pp. 523-528.

[16] S. Asmussen, L. Rojas-Nandayapa, "Sums of dependent lognormal random variables: asymptotics and simulation", Dec. 2005.

[17] B. G. Lindsay, P. Basak, "Moments determine the tail of a distribution (but not much else)," The American Statistician, vol. 54, Nov. 2004.

[18] M. A. Lifshits, "On the lower tail probabilities of some random series," The Annals of Probability, vol. 25, 1997, pp. 424-442.

[19] N. M. Temme, "Uniform asymptotic expansions of the incomplete gamma functions and the incomplete beta function," Mathematics of Computation, vol. 29, Oct. 1975, pp. 1109-1114.

[20] H. E. Fettis, "A stable algorithm for computing the inverse error function in the "Tail-End" Region," Mathematics of Computation, vol. 28, Apr. 1974, pp. 585-587.

TABLE I

COMPARISON OF THE FOUR METHODS

\begin{tabular}{lllll}
\hline \hline Form & $\begin{array}{c}\text { Includes } \\
\text { lognormal }\end{array}$ & $\begin{array}{c}\text { Number of } \\
\text { parameters }\end{array}$ & $\begin{array}{c}\text { Applies to } \\
\text { non-i.i.d. }\end{array}$ & $\begin{array}{c}\text { Lognormal } \\
\text { tails }\end{array}$ \\
\hline BR & Limit case & 3 & No & \\
LL & No & 3 & Yes & No \\
ZD & Yes & 3 & No & \\
ZS & No & 4 & Yes & \\
\hline \hline
\end{tabular}

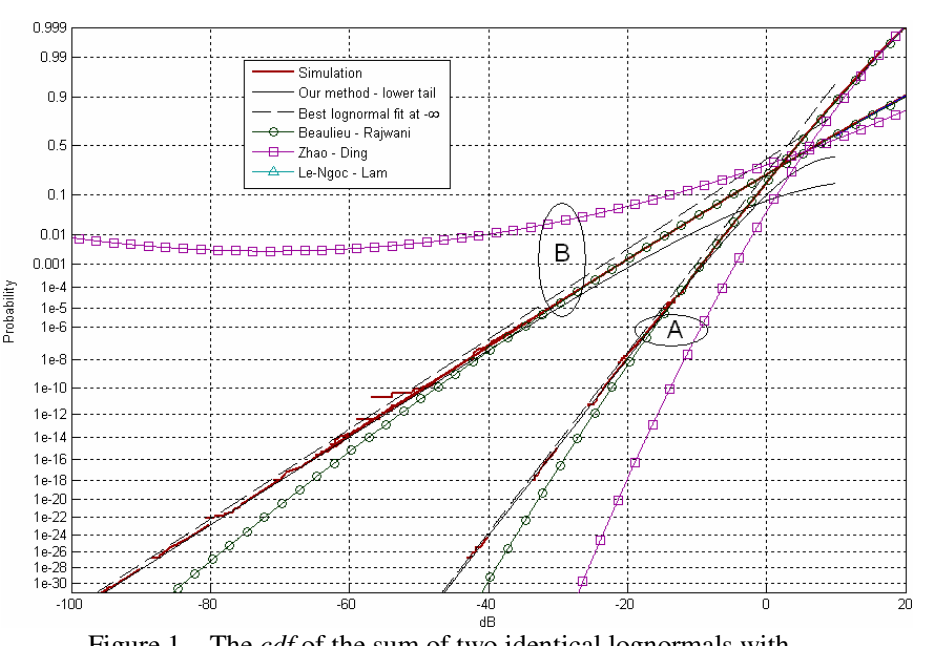

Figure 1. The $c d f$ of the sum of two identical lognormals with A: $\sigma=6 \mathrm{~dB}$ and B: $\sigma=12 \mathrm{~dB}$.

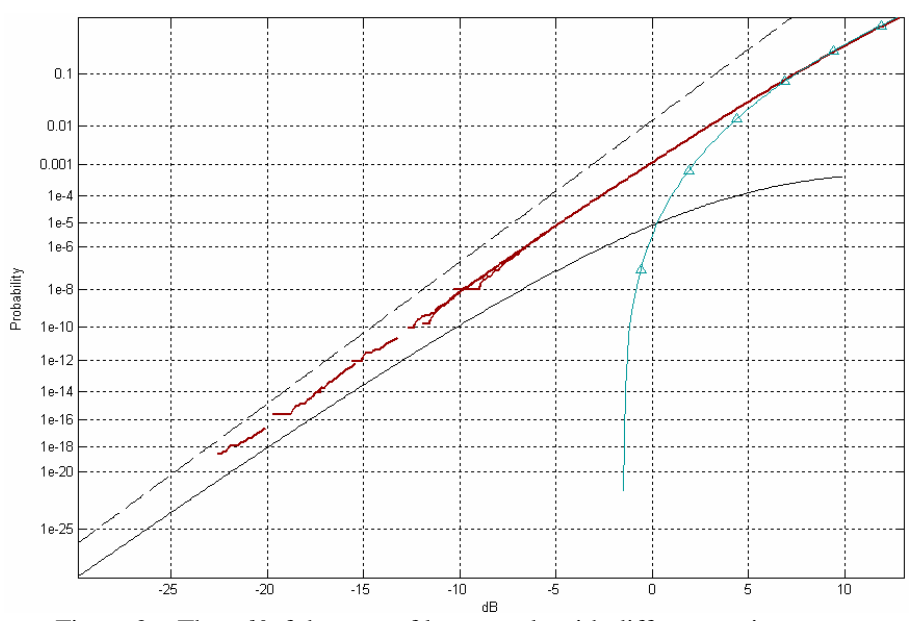

Figure 2. The $c d f$ of the sum of lognormals with different variances.

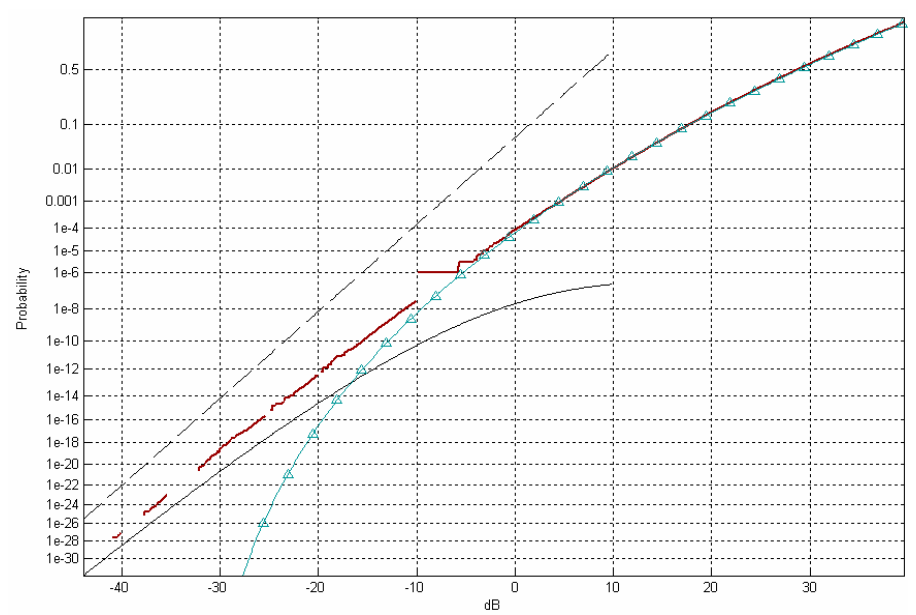

Figure 3. The $c d f$ of the sum of lognormals with different means.

TABLE II

FIGURE PARAMETERS

\begin{tabular}{lllll}
\hline \hline Figure & $N$ & & \multicolumn{1}{c}{$\mu(\mathrm{dB})$} & \multicolumn{1}{c}{$\sigma(\mathrm{dB})$} \\
\hline $1-\mathrm{A}$ & 2 & 0 & 6 \\
$1-\mathrm{B}$ & 2 & 0 & 12 \\
2 & 6 & 0 & $6,8,9,10,11,12$ \\
3 & 6 & $-25,-15,-5,5,15,25$ & 12 \\
\hline \hline
\end{tabular}

